\title{
Anti-inflammatory effects of dihydromyricetin in a mouse model of asthma
}

\author{
BIN XU* ${ }^{*}$, SHURAN HUANG ${ }^{*}$, CAIYING WANG, HAITAO ZHANG, SHENGCUN FANG and YINGMING ZHANG \\ Department of Respiratory Medicine, Nanjing Chest Hospital, Nanjing, Jiangsu 210029, P.R. China
}

Received February 19, 2016; Accepted February 1, 2017

DOI: $10.3892 / \mathrm{mmr} .2017 .6428$

\begin{abstract}
Dihydromyricetin (DHM) is a plant flavonoid and is the primary active ingredient isolated from the medicinal herb, Ampelopsis grossedentata. DHM has been shown to possess various pharmacological activities, including anti-inflammatory effects. However, the possible role of DHM in asthma treatment remains to be elucidated. The present study aimed to investigate its anti-inflammatory properties in mice with symptoms of allergic asthma. The C57BL/6 mice were sensitized and challenged with ovalbumin (OVA) to induce asthma. DHM or phosphate-buffered saline treatment was administered $1 \mathrm{~h}$ prior to the OVA challenge. The levels of interleukin (IL)-4, IL-5 and IL-13 in the bronchoalveolar lavage (BAL) fluid were measured by enzyme-linked immunosorbent assay (ELISA), and OVA-specific serum IgE and IgG1 levels were also determined by ELISA. Histopathological staining was performed to evaluate the infiltration of inflammatory cells into the BAL fluid, lung tissues and goblet cell hyperplasia. DHM treatment significantly reduced the total number of inflammatory cells, including eosinophils, neutrophils, lymphocytes and macrophages, in the BAL fluid. DHM also reduced the levels of IL-4, IL-5 and IL-13 in the BAL fluid, and reduced the secretion of OVA-specific $\operatorname{IgE}$ and $\operatorname{IgG} 1$ in the serum. The histological staining demonstrated that DHM treatment effectively suppressed the OVA-induced inflammatory cells in
\end{abstract}

Correspondence to: Dr Yingming Zhang, Department of Respiratory Medicine, Nanjing Chest Hospital, 215 Guangzhou Road, Nanjing, Jiangsu 210029, P.R. China

E-mail: zym_yingming@sina.com

*Contributed equally

Abbreviations: DHM, dihydromyricetin; OVA, ovalbumin; PBS, phosphate-buffered saline; Dex, dexamethasone; ELISA, enzyme-linked immunosorbent assay; BAL, bronchoalveolar lavage; NF- $\kappa \mathrm{B}$, nuclear factor $\kappa \mathrm{B}$; AHR, airway hyperresponsiveness; MAPKs, mitogen-activated protein kinases

Key words: dihydromyricetin, asthma, cytokines, inflammation, ovalbumin the lung tissues and in the mucus hypersecreted by goblet cells in the airway. These results showed that DHM had a potent anti-inflammatory effect in an OVA-induced mouse model of asthma, offering potential as an anti-inflammatory agent for the treatment of asthma.

\section{Introduction}

Asthma is one of the most common chronic respiratory diseases, characterized by variable airflow obstruction, airway inflammation and remodeling, bronchial hyperresponsiveness and mucus hypersecretion, excessive infiltration of leukocytes (particularly eosinophils) into the airways, and intrapulmonary overexpression of Th2 cytokines, including interleukin (IL)-4, IL-5 and IL-13 (1,2). Increasing evidence has indicated that the incidence of asthma has markedly increased worldwide, and its morbidity and mortality rates have increased in previous decades, despite advances in understanding its pathophysiological mechanism $(3,4)$. In addition, although asthma treatment has improved by the implementation of management guidelines, and the use of inhaled corticosteroids and $\beta-2$ agonists as the first line treatment for asthmatics (5), current treatments and the well-known side effects of corticosteroids are not always satisfactory in clinical practice (6). Therefore, novel or alternative approaches for the treatment of asthma are potentially useful and increasingly critical.

Dihydromyricetin (DHM) is a plant flavonoid component isolated from the tender stem and leaves of Ampelopsis grossedentata, which is widely used in traditional Chinese medicine in South China for preventing and treating hepatitis, chronic nephritis, vomiting, acid regurgitation, polyorexia and halitosis $(7,8)$. The chemical structure of DHM is shown in Fig. 1A, which is similar to that of myrecetin, a natural flavonoid found in berries, grapes, vegetables, herbs, fruits and other plants with anticancer activities $(9,10)$. Previous studies have indicated that DHM exhibits a range of biological and pharmacological activities, including hepatic protection $(11,12)$, attenuation of cardiomyocyte hypertrophy (13), antimicrobial activity, anti-oxidation and antitumor effects (14-17).

In previous years, the anti-inflammatory effect of DHM has attracted increased interest. DHM supplementation has been found to exert anti-inflammatory activity in patients with nonalcoholic fatty liver disease through decreased levels of serum tumor necrosis factor- $\alpha$, cytokeratin-18 and 
fibroblast growth factor 21 (18). Additionally, studies have suggested that DHM possesses anti-inflammatory effects via inhibiting the activity of nuclear factor $\kappa \mathrm{B}(\mathrm{NF}-\kappa \mathrm{B})$ and mitogen-activated protein kinase (MAPK) signaling pathways $(19,20)$. However, no information is currently available on the effect of DHM treatment on a non-infectious mouse airway inflammatory model. Therefore, in the present study, an ovalbumin (OVA) -induced asthmatic model was used to evaluate whether DHM has a distinct anti-inflammatory role in asthmatic mice.

\section{Materials and methods}

Preparation of DHM. DHM was purchased from SigmaAldrich; Merck KGaA (Darmstadt, Germany), dissolved in ethanol and prepared as a $10 \mathrm{mg} / \mathrm{ml}$ stock solution, which was diluted to the final concentration with sterile phosphatebuffered saline (PBS; pH 7.4) immediately prior to use.

Animals. Female C57BL/6 mice (4-6 weeks old) were purchased from Beijing Biocytogen Company (Beijing, China), and maintained in a pathogen-free animal room at a constant temperature $\left(25^{\circ} \mathrm{C}\right)$ in $12: 12$-h light:dark cycle with access to water and standard chow ad libitum. The guidelines of the Animal Care and Use Committee (ACUC) of Nanjing Chest Hospital (Nanjing, China) were adhered to in strict accordance, and the experimental procedures were reviewed and approved by the ACUC.

Sensitization and treatment protocols. The mice were divided into four groups, each containing eight mice: Negative control group; OVA group, OVA+dexamethasone (Dex; Sigma-Aldrich; Merck KGaA) group; and OVA+DHM group. The induction of asthma in the mice treated with OVA (grade Ш) was performed as described previously (21). Briefly, each mouse was sensitized with $20 \mu \mathrm{g}$ of OVA in $1 \mathrm{mg}$ aluminum hydroxide by intraperitoneal injection on days 0,7 and 14. On days 21-27, the OVA-challenged mice were exposed to inhalation with 5\% OVA solution, which was aerosolized using an ultrasonic nebulizer for $1 \mathrm{~h}$ per day. The mice in the negative control groups were sensitized and challenged with an equal volume of PBS, instead of the OVA and drug treatment. The other three groups of asthma-induced mice were administered intraperitoneally with $10 \mathrm{mg} / \mathrm{kg}$ of DHM, based on preliminary experiments and a previous study (6), or $2 \mathrm{mg} / \mathrm{kg}$ of OVA as a positive control between days 14 and 27.

Collection of bronchoalveolar lavage (BAL) fluid. Following the final challenge, the mice were sacrificed and the thoracic cavity was immediately opened by careful dissection. The trachea was then exposed, and BAL fluid was collected by lavage of the lung through the trachea infused with three aliquots of PBS $(0.3,0.3$ and $0.4 \mathrm{ml})$ in a total volume of $1 \mathrm{ml}$. The fluids were then centrifuged at $400 \mathrm{x} g$ for $5 \mathrm{~min}$ at $4^{\circ} \mathrm{C}$, and the pellets were recovered for cellular analysis. The supernatants were stored at $-80^{\circ} \mathrm{C}$ until biochemical analyses.

Counts of inflammatory cells in BAL fluid. The cell pellets were resuspended in $500 \mu 1$ of PBS, and the total number of viable cells were counted using a hemocytometer through Trypan blue exclusion. Cytological examination was performed as described previously (22). Briefly, cytospin preparations were produced using a Cytospin (Thermo Fisher Scientific, Inc., Waltham, MA, USA) and stained with modified Wright's stain. Differential cell counting was performed on at least 200 cells in each slide using standard morphological criteria under a light microscope (CX41; Olympus Corporation, Tokyo, Japan).

ELISA. The expression levels of OVA-specific IgE and IgG1 in the mouse sera and cytokines in the BAL fluid were measured using the ELISA method, according to a previously described method (2) with modifications. Briefly, blood samples were immediately collected from murine orbita following sacrifice of the mice on day 28 , followed by centrifugation at $1,000 \times \mathrm{g}$ for $15 \mathrm{~min}$ at $4^{\circ} \mathrm{C}$. Aliquots of the serum were then stored at $-80^{\circ} \mathrm{C}$ until analysis. For OVA-specific IgE and IgG1 analysis, microplate wells were respectively coated with purified rat anti-mouse IgE (ab99574; 1:300; Abcam, Cambridge, UK) and IgG1 (ab99656; 1:300; Abcam), followed by incubation with blocking buffer at room temperature for $1 \mathrm{~h}$ and subsequent washing with PBS. Subsequently, mouse sera were added to the microplate wells and incubated for $2 \mathrm{~h}$ at room temperature, followed by incubation with biotin-labeled anti-mouse $\mathrm{IgE}$ or IgG1 for $1 \mathrm{~h}$ at room temperature. The reactions were read using an automated spectrophotometric plate reader (PerkinElmer, Inc., Waltham, MA, USA) at $450 \mathrm{~nm}$. The levels of IL-4, IL-5 and IL-13 in the BAL fluid were also determined using corresponding specific ELISA kits (BD Biosciences, Franklin Lakes, NJ, USA).

Lung tissue histopathology. Following collection of BAL fluid, lung tissues were excised from the mice and fixed in $10 \%$ (v/v) formalin overnight. The tissue was then embedded in paraffin and cut into sections of $5 \mu \mathrm{m}$ thickness, followed by deparaffinization and rehydration. The sections were then stained with hematoxylin and eosin (H\&E) for detecting the infiltration of inflammatory cells, and stained with periodic acid-Schiff (PAS) staining to measure mucus production under a light microscope (CX41; Olympus Corporation).

Measurement of airway hyperresponsiveness (AHR). AHR was measured as described previously (23). Briefly, $24 \mathrm{~h}$ following the final OVA challenge, conscious and unrestrained mice were used to evaluate AHR using whole-body plethysmography (Buxco Electronics, Inc., Wilmington, NC, USA). Each mouse was placed in a single chamber and subjected to exposure of aerosolized PBS, followed by 5,10 , 20 and $40 \mathrm{mg} / \mathrm{ml}$ solutions of aerosolized methacholine in PBS for 3 min each time $(n=4)$, and the interval duration between each exposure was $30 \mathrm{~min}$. Following each exposure of methacholine at different concentrations, bronchoconstriction was recorded using whole-body plethysmography. The results were calculated as the proportion of the respective basal Penh values obtained from the PBS-challenged control mice.

Statistical analysis. Data are expressed as the mean \pm standard deviation. Statistical analysis was performed using the SPSS 
A

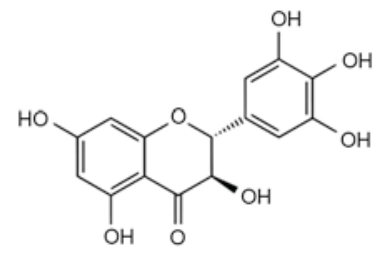

Chemical structure of dihydromyricetin

B

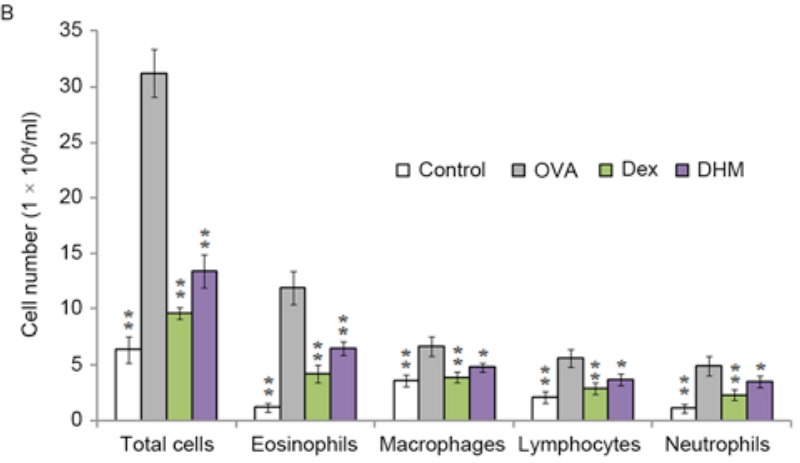

Figure 1. Effect of DHM on OVA-induced inflammatory cells counts in BAL fluid. (A) Chemical structure of DHM. (B) Recovered cells from BAL fluid were counted following Wright's staining. Data are expressed as the mean \pm standard deviation $(n=8),{ }^{*} \mathrm{P}<0.05$ and ${ }^{* *} \mathrm{P}<0.01$, vs. OVA. DHM, dihydromyricetin; OVA, ovalbumin; BAL, bronchoalveolar lavage.

A

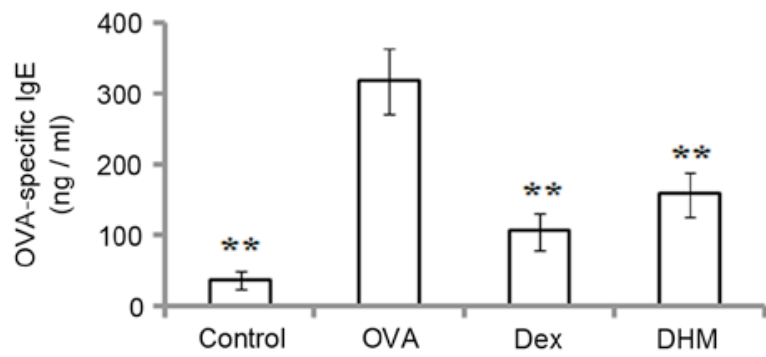

B

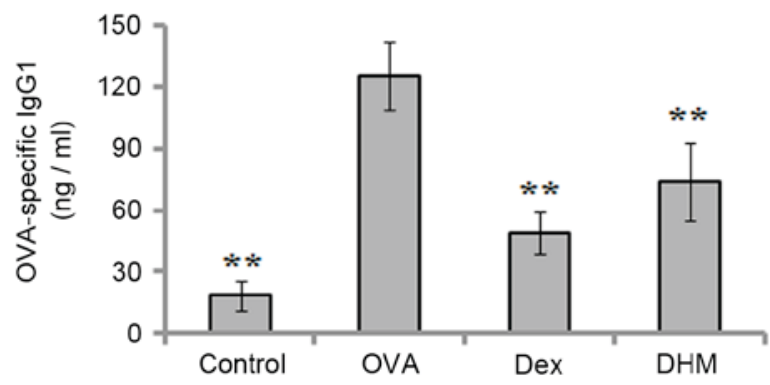

Figure 2. Effects of DHM on OVA-induced IgE and IgG1 in serum. Mouse serum was collected $24 \mathrm{~h}$ following the final OVA challenged and the levels of OVA-specific (A) IgE and (B) IgG1 in serum were determined using enzyme-linked immunosorbent assays. Data are expressed as the mean \pm standard deviation $(\mathrm{n}=8),{ }^{* * *} \mathrm{P}<0.01$, vs. OVA. DHM, dihydromyricetin; OVA, ovalbumin; Dex, dexamethasone

statistical software package (version 11.0; SPSS, Inc., Chicago, IL, USA) using Student's two-tail $t$-test. $\mathrm{P}<0.05$ was considered to indicate a statistically significant difference.

\section{Results}

Effect of DHM on the infiltration of inflammatory cells in $B A L$ fluid. Alterations in cellular components, particularly eosinophils, are critical in the development of asthma. To evaluate the anti-inflammatory effects of DHM, inflammatory cells, including eosinophils, macrophages, lymphocytes and neutrophils, in the BAL fluid of mice challenged with OVA were stained with Wright's stain and counted. The results demonstrated that OVA challenge resulted in an apparent increased influx of eosinophils, macrophages, lymphocytes and neutrophils into the BAL fluid of the OVA-sensitized mice. However, DHM challenge significantly reduced the number of these inflammatory cells in the BAL fluid, compared with OVA-challenged mice (Fig. 1B).

Effect of DHM on the release of OVA-specific IgE and IgGI into serum. In the pathogenesis of the allergic response, IgE and IgG1 are associated with inflammatory cells. Therefore, the levels of OVA-specific IgE and IgG1 in the serum were determined using ELISA. As shown in Fig. 2, the results demonstrated that the levels of IgE (Fig. 2A) and IgG1 (Fig. 2B) in the serum were markedly increased in the OVA-treated mice, compared with the control mice. However, DHM treatment significantly decreased the OVA-specific levels of $\operatorname{IgE}$ and IgG1, compared with the OVA-challenged group.

Effect of DHM on cytokine levels. To determine the effect of DHM on the release of cytokines in OVA-challenged mice, the levels of IL-4, IL-5 and IL-13 in the BAL fluid were measured using ELISA $24 \mathrm{~h}$ following the final challenge. As shown in Fig. 3, the results demonstrated that OVA sensitization and challenge resulted in marked elevations in the production of IL-4, IL-5 and IL-13 in the BAL fluid of the mice, however, these elevated levels of IL-4 (Fig. 3A), IL-5 (Fig. 3B) and IL-13 (Fig. 3C) were significantly downregulated by treatment with DHM and the positive control, Dex.

Effect of DHM on OVA-induced inflammatory cells in lung tissue. To evaluate the anti-inflammatory effect of DHM, asthmatic lung tissue was collected from the mice $24 \mathrm{~h}$ following the final OVA challenge, and stained with H\&E solution. The results showed that no inflammatory cell infiltration was observed in the normal lung tissue of the control mice (Fig. 4A). By contrast, in the OVA-challenged mice, the infiltration of inflammatory cells, which were predominantly eosinophils, were found in the peribronchial and perivascular areas of the lung tissue (Fig. 4B). However, DHM treatment and positive control (Dex) treatment effectively inhibited the infiltration of inflammatory cells into the lung tissues of the OVA-challenged mice (Fig. 4C-D).

Effect of DHM on airway goblet cell hyperplasia and mucus production. To evaluate the effect of DHM on mucus overproduction caused by goblet cell hyperplasia, lung tissue sections were stained with PAS staining. As shown in Fig. 5, the results demonstrated that mucus hypersecretion and goblet cell hyperplasia were observed in the bronchi of the airways in the OVA-challenged mice, but not in the control mice (Fig. 5A and B). However, mucus production and the number of goblet 
A
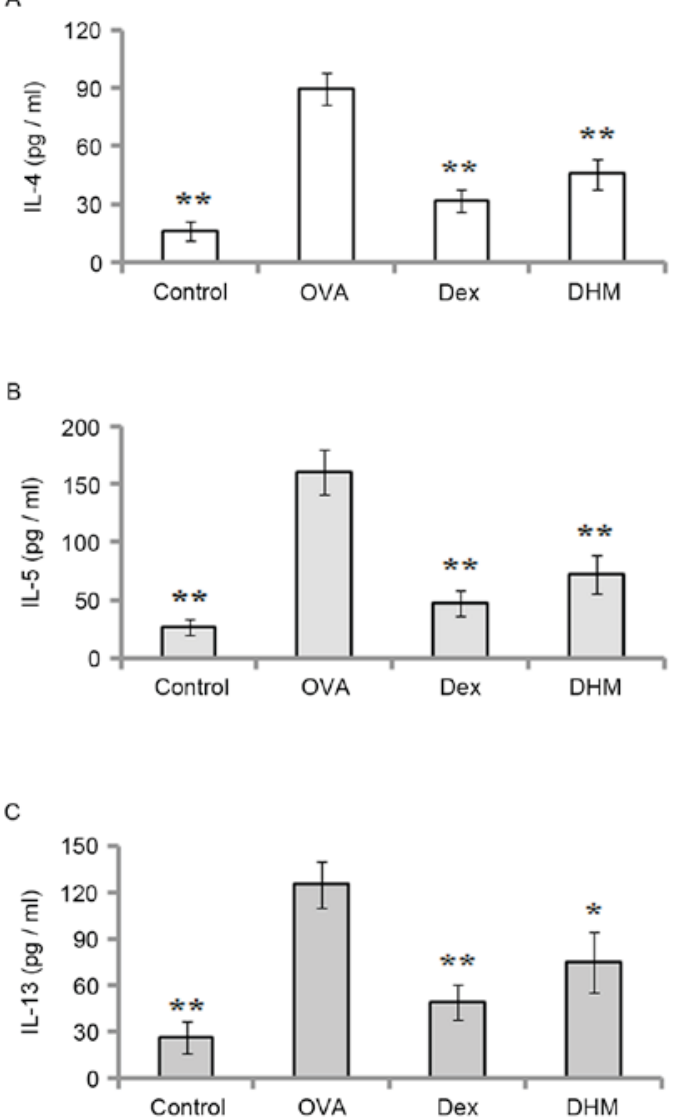

Figure 3. Effects of DHM on cytokine levels in BAL fluid. BAL fluid was collected following the final OVA challenge, and the levels of (A) IL-4, (B) IL-5 and (C) IL-13 were analyzed using enzyme-linked immunosorbent assays. Data are expressed as the mean \pm standard deviation $(\mathrm{n}=8),{ }^{*} \mathrm{P}<0.05$ and ${ }^{* *} \mathrm{P}<0.01$, vs. OVA. DHM, dihydromyricetin; OVA, ovalbumin; Dex, dexamethasone; IL, interleukin.
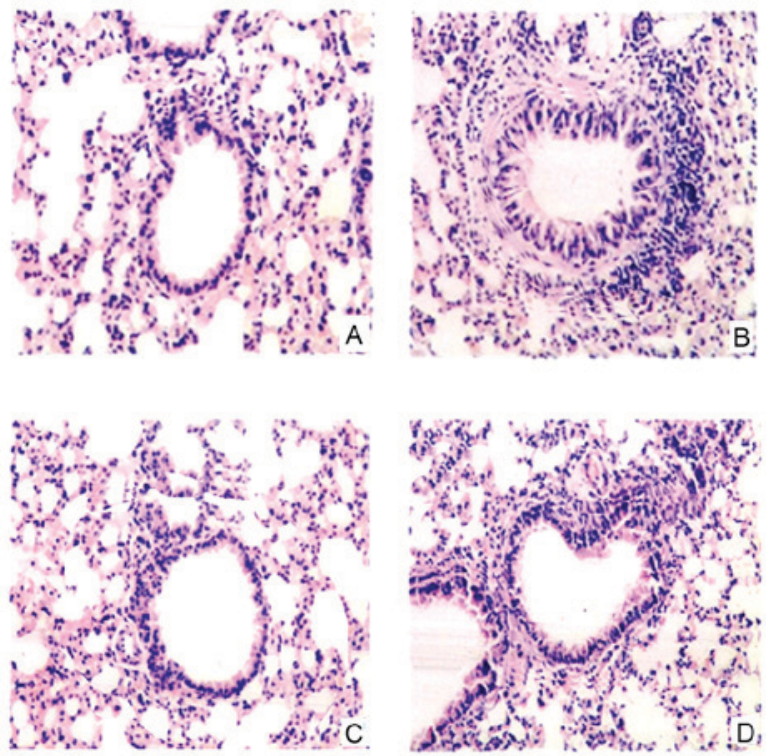

Figure 4. Effect of DHM on the recruitment of inflammatory cells in lung tissue. Lung tissues were obtained from the mice following the final OVA challenge, and was fixed, embedded, cut into sections and stained with hematoxylin and eosin solution to detect the infiltration of inflammatory cells (A) phosphate-buffered saline-challenged mice; (B) OVA-challenged mice; (C) asthmatic mice treated with dexamethasone (2 mg/kg); (D) asthmatic mice treated with DHM $(10 \mathrm{mg} / \mathrm{kg})$. Magnification, x200. DHM, dihydromyricetin; OVA, ovalbumin.
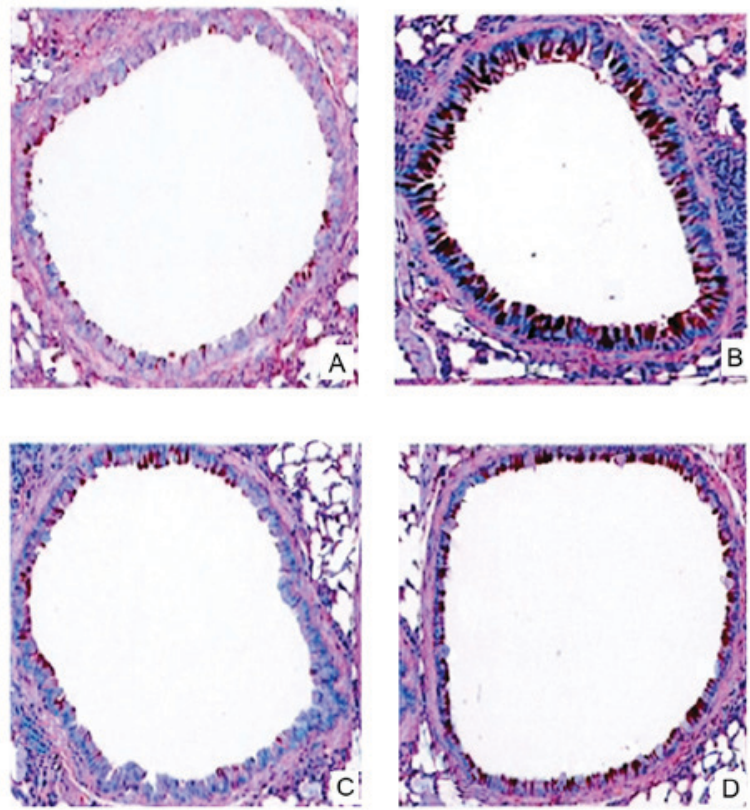

Figure 5. Effect of DHM on mucus production. Lung tissues were fixed, embedded, cut into section, and then stained with periodic acid-Schiff solution to identify mucus hypersecretion. (A) Phosphate-buffered saline-challenged mice; (B) ovalbumin-challenged mice; (C) asthmatic mice treated with dexamethasone $(2 \mathrm{mg} / \mathrm{kg})$; (D) asthmatic mice treated with DHM $(10 \mathrm{mg} / \mathrm{kg})$. Magnification, x400. DHM, dihydromyricetin.

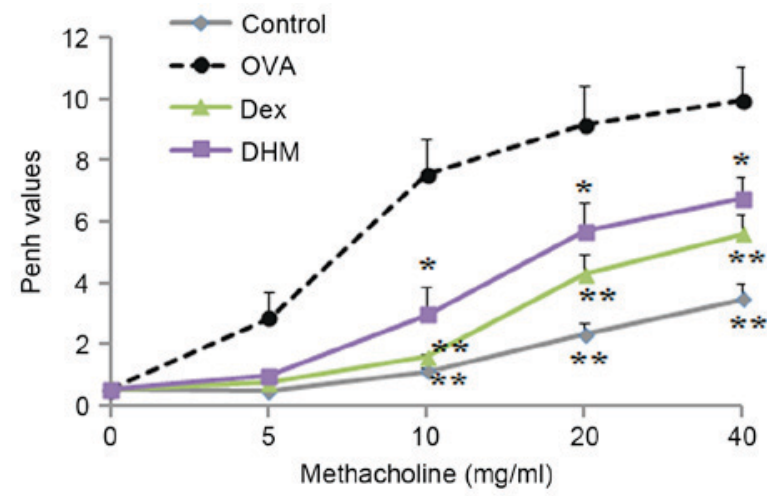

Figure 6. Effect of DHM on AHR. AHR in the mice treated with increasing concentrations of methacholine $(0-40 \mathrm{mg} / \mathrm{ml})$ was measured using whole-body plethysmography $24 \mathrm{~h}$ following the final OVA challenge. Penh values are expressed as mean \pm standard deviation $(n=5)$. ${ }^{*} \mathrm{P}<0.05$ and ${ }^{* *} \mathrm{P}<0.01$, vs. OVA. DHM, dihydromyricetin; OVA, ovalbumin; Dex, dexamethasone; AHR, airway hyperresponsiveness.

cells were markedly decreased in the Dex-treated (Fig. 5C) and DHM-treated (Fig. 5D) asthmatic mice.

Effect of DHM on AHR. To determine the inhibitory effect of DHM on AHR, the OVA-challenged mouse model, in which methacholine served as an AHR promoter, was used. AHR was calculated by Penh values (enhanced pauses). The results showed that, in the OVA-challenged mice, the Penh value was higher, compared with that of control group when the concentration of methacholine increased between 5 and $40 \mathrm{mg} / \mathrm{ml}$. Treatment with the positive control (Dex) or DHM significantly decreased the Penh value, compared with that in 
the OVA-challenged group at a methacholine concentration range of $10-40 \mathrm{mg} / \mathrm{ml}$ (Fig. 6).

\section{Discussion}

Asthma is one of the most common inflammatory diseases of the airways, characterized by increased infiltration of various inflammatory cells into the lung airways, bronchial hyperresponsiveness, reversible airway obstruction and reduced respiratory function (24). Due to the prevalence of asthma worldwide and the various side effects resulting from the long-term use of corticosteroids and $\beta-2$ agonists, it is necessary to investigate effective therapeutic agents for the treatment of asthma. DHM is a major bioactive flavonoid, originally isolated from the medical herb, Ampelopsis grossedentata, which has been found to possess various pharmacological activities (8). However, the effect of DHM treatment on asthma remains to be elucidated. In the present study, the anti-inflammatory effect of DHM in an OVA-induced asthmatic mouse model was investigated. It was found that DHM effectively suppressed the infiltration of inflammatory cells into the airways of the asthmatic mice, decreased the numbers of eosinophils, macrophages, lymphocytes and neutrophils into the BAL fluid, and inhibited the elevated levels of IL-4, IL-5 and IL-13 in the BAL fluid. In addition, reduction in the levels of OVA-specific IgE and IgG1 was observed in the serum of mice challenged with DHM. The present study is the first, to the best of our knowledge, to evaluate the effects of DHM in a non-infectious mouse model of asthma.

Previous studies have indicated that asthma was originally considered to be a Th2-mediated disease (25). Accordingly, Th2 cytokines have distinct and significant effects in the pathogenesis of asthma. Their overproduction induces the inflammatory response and airway remodeling, and contributes to the initiation and pathogenesis of acute asthma (26). Therefore, cytokines have become a major target of novel therapies for asthma. In the present study, it was found that the levels of IL-4, IL-5 and IL-13 in the BAL fluid were markedly elevated in OVA-challenged mice, but were decreased following DHM treatment. In a previous study, DHM was demonstrated to possess anti-inflammatory activity through reducing the production of tumor necrosis factor- $\alpha$ and cytokeratin-18 in patients with non-alcoholic fatty liver disease (18). Proinflammatory cytokines, including IL-4, IL-5 and IL-13, expressed by Th2 cells are directly involved in the initiation and maintenance of allergic disease (27). IL-4 may be responsible for the production of $\operatorname{IgE}(28)$. IL-5 promotes the mobilization of eosinophils from the bone marrow, and recruits eosinophils into the airways (29). IL-13 is important in eosinophilic inflammation, mucus hypersecretion and airway luminal narrowing (30). The data in the present study suggested that DHM treatment decreased the levels of IL-4, IL-5 and IL-13 in the OVA-induced asthmatic mouse, which at least contributed to the anti-inflammatory effect of DHM.

Eosinophilia is a major hallmark of allergic diseases. Eosinophils are the predominant inflammatory cells in asthmatic lung tissues, and are known principal effector cells in the pathogenesis of allergic inflammation (31). The recruitment of eosinophils into the airways is a multistep process, which is coordinated by various cytokines and adhesion molecules (32). The results of the present study demonstrated that DHM treatment effectively reduced the number of eosinophils in the BAL fluid of the OVA-challenged mice. Other leukocytes, including neutrophils, macrophages and lymphocytes, are also important mediators for the inflammatory response. In the present study, DHM treatment ameliorated airway eosinophilia, as shown by the decreased numbers of eosinophils, macrophages, lymphocytes and neutrophils in the BAL fluid and lung tissues of the OVA-sensitized mice. Additionally, the findings demonstrated that DHM treatment significantly decreased mucus hypersecretion. A previous study indicated that mucus hypersecretion was increased by hypertrophy of goblet cells in the lung tissue of asthma, which is involved in airway obstruction (33).

Increased levels of serum IgE and IgG1 are a hallmark of the Th2 immune response, and it generally observed in OVA-challenged animals, similar to the increase in pollen-specific IgE in hayfever sufferers (34). The biological activity of $\mathrm{IgE}$ is mediated through its interaction with its high affinity IgE receptor on mast cells and basophils (22). It has been reported that the neutralization of $\operatorname{IgE}$ decreased the recruitment of eosinophils into the airway following allergen challenge (35). In the present study, DHM treatment inhibited the increased serum production of $\operatorname{IgE}$ and $\mathrm{IgG1}$, which suggested that the anti-allergy effect of DHM was associated with the decreased production of $\operatorname{IgE}$ and $\mathrm{IgG1}$.

AHR is a useful marker of airway abnormality in asthma, and its development involves various inflammatory cells and mediators. The data obtained in the present study demonstrated that DHM significantly suppressed the OVA-induced AHR to inhaled methacholine. IL-5-mediated eosinophilia is important in AHR development by generating various proinflammatory products, including cysteinyl-leukotrienes, major basic protein, eosinophilic cationic protein and other lipid mediators, which are closely associated with AHR (36). Additionally, IL-4 and IL-13 have been reported to induce AHR in mouse asthma models (37). Thus, the DHM-mediated reduction of AHR may be associated with reduction in the production of IL-4, IL-5 and IL-13.

Previous studies have indicated that the production of several inflammatory cytokines is regulated by MAPKs and $\mathrm{NF}-\kappa \mathrm{B}(38-40)$. The MAPK signaling cascade is important in the activation of inflammatory cells. Suppression of the extracellular signal-regulated kinase (ERK) signaling pathway may be a therapeutic target for the treatment of allergic airway inflammation (4). In addition, when $N F-\kappa B$ is activated, several inflammatory cytokines are upregulated in asthmatic airways, and the AHR and mucus secretion are enhanced in allergic inflammation (41). Previous studies have suggested that DHM inhibits the activation of NF- $\kappa \mathrm{B}$ and the phosphorylation of p38 and c-Jun N-terminal kinase, but not ERK1/2, in lipopolysaccharide-stimulated macrophages $(18,20,42)$. In the present study, it was hypothesized that DHM inhibited the inflammatory response in the asthma model through a similar mechanism; however, this requires clarification in future investigations.

In conclusion, the present study showed that DHM treatment effectively inhibited the increased number of inflammatory 
cells, including eosinophils, macrophages, lymphocytes and neutrophils, in the mouse asthma model induced by OVA, and also effectively suppressed the elevated levels of $\mathrm{IgE}$ and IgG1, and of the IL-4, IL-5 and IL-13 cytokines in the OVA-challenged mice. Additionally, histological staining indicated that DHM treatment markedly inhibited the infiltration of inflammatory cells in the lung tissue and reduced mucus hypersecretion by goblet cells in the airway. These findings suggested that DHM may have inhibitory effects on airway inflammation and may be a potential therapeutic agent for the treatment of asthma.

\section{Acknowledgements}

The authors would like to thank Dr Joel Thomson at the Foreign Language Institute in Guangdong Medical College (Zhanjiang, China) for proofreading the English of this manuscript.

\section{References}

1. Deveci F, Muz MH, Ilhan N, Kirkil G, Turgut T and Akpolat N: Evaluation of the anti-inflammatory effect of infliximab in a mouse model of acute asthma. Respirology 13: 488-497, 2008.

2. Ci X, Chu X, Xiang H, Li X and Deng X: Anti-inflammatory effects of tilmicosin in a noninfectious mouse model of allergic asthma. Immunopharmacol Immunotoxicol 33: 626-632, 2011.

3. Munroe ME, Businga TR, Kline JN and Bishop GA: Anti-inflammatory effects of the neurotransmitter agonist Honokiol in a mouse model of allergic asthma. J Immunol 185: 5586-5597, 2010

4. Lee SH, Kim DW, Kim HR, Woo SJ, Kim SM, Jo HS, Jeon SG, Cho SW, Park JH, Won MH, et al: Anti-inflammatory effects of Tat-Annexin protein on ovalbumin-induced airway inflammation in a mouse model of asthma. Biochem Biophys Res Commun 417: 1024-1029, 2012.

5. Murad HA and Hasanin AH: The anti-inflammatory effects of 1,1 dimethyl-4-phenylpiperazinium (DMPP) compared to dexamethasone in a guinea pig model of ovalbumin induced asthma. Eur Rev Med Pharmacol Sci 18: 2228-2236, 2014.

6. Kim DY, Park BS, Hong GU, Lee BJ, Park JW, Kim SY and Ro JY: Anti-inflammatory effects of the R2 peptide, an inhibitor of transglutaminase 2 , in a mouse model of allergic asthma, induced by ovalbumin. Br J Pharmacol 162: 210-225, 2011.

7. Zeng G, Liu J, Chen H, Liu B, Zhang Q, Li M and Zhu R: Dihydromyricetin induces cell cycle arrest and apoptosis in melanoma SK-MEL-28 cells. Oncol Rep 31: 2713-2719, 2014.

8. Shen Y, Lindemeyer AK, Gonzalez C, Shao XM, Spigelman I, Olsen RW and Liang J: Dihydromyricetin as a novel anti-alcohol intoxication medication. J Neurosci 32: 390-401, 2012.

9. Ni F, Gong Y, Li L, Abdolmaleky HM and Zhou JR: Flavonoid ampelopsin inhibits the growth and metastasis of prostate cancer in vitro and in mice. PLoS One 7: e38802, 2012.

10. Huang H, Hu M, Zhao R, Li P and Li M: Dihydromyricetin suppresses the proliferation of hepatocellular carcinoma cells by inducing G2/M arrest through the Chk1/Chk2/Cdc25C pathway. Oncol Rep 30: 2467-2475, 2013.

11. Murakami T, Miyakoshi M, Araho D, Mizutani K, Kambara T, Ikeda T, Chou WH, Inukai M, Takenaka A and Igarashi K: Hepatoprotective activity of tocha, the stems and leaves of Ampelopsis grossedentata, and ampelopsin. Biofactors 21: 175-178, 2004.

12. Liang J, Shen Y, Shao XM, Scott MB, Ly E, Wong S, Nguyen A, Tan K, Kwon B, Olsen RW and Spigelman I: Dihydromyricetin prevents fetal alcohol exposure-induced behavioral and physiological deficits: The roles of GABAA receptors in adolescence. Neurochem Res 39: 1147-1161, 2014.

13. Meng G, Yang S, Chen Y, Yao W, Zhu H and Zhang W: Attenuating effects of dihydromyricetin on angiotensin II-induced rat cardiomyocyte hypertrophy related to antioxidative activity in a NO-dependent manner. Pharm Biol 53: 904-912, 2015.
14. Jiang B, Le L, Pan H, Hu K, Xu L and Xiao P: Dihydromyricetin ameliorates the oxidative stress response induced by methylglyoxal via the AMPK/GLUT4 signaling pathway in PC12 cells. Brain Res Bull 109: 117-126, 2014.

15. Liao W, Ning Z, Ma L, Yin X, Wei Q, Yuan E, Yang J and Ren J: Recrystallization of dihydromyricetin from Ampelopsis grossedentata and its anti-oxidant activity evaluation. Rejuvenation Res 17: 422-429, 2014.

16. Zhao Z, Yin JQ, Wu MS, Song G, Xie XB, Zou C, Tang Q, Wu Y, Lu J, Wang Y, et al: Dihydromyricetin activates AMP-activated protein kinase and P38(MAPK) exerting antitumor potential in osteosarcoma. Cancer Prev Res 7: 927-938, 2014.

17. Zhu H, Luo P, Fu Y, Wang J, Dai J, Shao J, Yang X, Chang L, Weng Q, Yang B and He Q: Dihydromyricetin prevents cardiotoxicity and enhances anticancer activity induced by adriamycin. Oncotarget 6: 3254-3267, 2015.

18. Chen S, Zhao X, Wan J, Ran L, Qin Y, Wang X, Gao Y, Shu F, Zhang Y, Liu P, et al: Dihydromyricetin improves glucose and lipid metabolism and exerts anti-inflammatory effects in nonalcoholic fatty liver disease: A randomized controlled trial. Pharmacol Res 99: 74-81, 2015.

19. Qi S, Xin Y, Guo Y, Diao Y, Kou X, Luo L and Yin Z: Ampelopsin reduces endotoxic inflammation via repressing ROS-mediated activation of $\mathrm{PI} 3 \mathrm{~K} / \mathrm{Akt} / \mathrm{NF}-\kappa \mathrm{B}$ signaling pathways. Int Immunopharmacol 12: 278-287, 2012.

20. Hou XL, Tong Q, Wang WQ, Shi CY, Xiong W, Chen J, Liu X and Fang JG: Suppression of inflammatory responses by dihydromyricetin, a flavonoid from ampelopsis grossedentata, via inhibiting the activation of NF- $\kappa \mathrm{B}$ and MAPK signaling pathways. J Nat Prod 78: 1689-1696, 2015.

21. Yang EJ, Lee JS, Yun CY, Ryang YS, Kim JB and Kim IS: Suppression of ovalbumin-induced airway inflammatory responses in a mouse model of asthma by Mimosa pudica extract. Phytother Res 25: 59-66, 2011.

22. Duan W, Chan JH, Wong CH, Leung BP and Wong WS: Anti-inflammatory effects of mitogen-activated protein kinase kinase inhibitor U0126 in an asthma mouse model. J Immunol 172: 7053-7059, 2004.

23. Lee M, Kim S, Kwon OK, Oh SR, Lee HK and Ahn K: Anti-inflammatory and anti-asthmatic effects of resveratrol, a polyphenolic stilbene, in a mouse model of allergic asthma. Int Immunopharmacol 9: 418-424, 2009.

24. Bisset LR and Schmid-Grendelmeier P: Chemokines and their receptors in the pathogenesis of allergic asthma: Progress and perspective. Curr Opin Pulm Med 11: 35-42, 2005.

25. Nakajima $\mathrm{H}$ and Takatsu K: Role of cytokines in allergic airway inflammation. Int Arch Allergy Immunol 142: 265-273, 2007

26. Ngoc PL, Gold DR, Tzianabos AO, Weiss ST and Celedón JC: Cytokines, allergy, and asthma. Curr Opin Allergy Clin Immunol 5: 161-166, 2005.

27. Elias JA, Lee CG, Zheng T, Ma B, Homer RJ and Zhu Z: New insights into the pathogenesis of asthma. J Clin Invest 111: 291-297, 2003.

28. Yan S, Ci X, Chen N, Chen C, Li X, Chu X, Li J and Deng X: Anti-inflammatory effects of ivermectin in mouse model of allergic asthma. Inflamm Res 60: 589-596, 2011.

29. Palmqvist C, Wardlaw AJ and Bradding P: Chemokines and their receptors as potential targets for the treatment of asthma. $\mathrm{Br} \mathrm{J}$ Pharmacol 151: 725-736, 2007.

30. Rosenberg HF, Phipps S and Foster PS: Eosinophil trafficking in allergy and asthma. J Allergy Clin Immunol 119: 1303-1312, 2007.

31. Kim DY, Ryu SY, Lim JY, Lee YS and Ro JY: Anti-inflammatory mechanism of simvastatin in mouse allergic asthma model. Eur J Pharmacol 557: 76-86, 2007.

32. Jia GQ, Gonzalo JA, Hidalgo A, Wagner D, Cybulsky M and Gutierrez-Ramos JC: Selective eosinophil transendothelial migration triggered by eotaxin via modulation of Mac-1/ICAM-1 and VLA-4/VCAM-1 interactions. Int Immunol 11: 1-10, 1999.

33. Woodruff PG and Fahy JV: A role for neutrophils in asthma? Am J Med 112: 498-500, 2002.

34. Wu AY, Chik SC, Chan AW, Li Z, Tsang KW and Li W: Anti-inflammatory effects of high-dose montelukast in an animal model of acute asthma. Clin Exp Allergy 33: 359-366, 2003.

35. Coyle AJ, Wagner K, Bertrand C, Tsuyuki S, Bews J and Heusser C: Central role of immunoglobulin (Ig) E in the induction of lung eosinophil infiltration and $\mathrm{T}$ helper 2 cell cytokine production: Inhibition by a non-anaphylactogenic anti-IgE antibody. J Exp Med 183: 1303-1310, 1996. 
36. Vargaftig BB and Singer M: Leukotrienes mediate murine bronchopulmonary hyperreactivity, inflammation, and part of mucosal metaplasia and tissue injury induced by recombinant murine interleukin-13. Am J Respir Cell Mol Biol 28: 410-419, 2003.

37. Taube C, Duez C, Cui ZH, Takeda K, Rha YH, Park JW, Balhorn A, Donaldson DD, Dakhama A and Gelfand EW: The role of IL-13 in established allergic airway disease. J Immunol 169: 6482-6489, 2002.

38. Inoue $\mathrm{H}$, Kato R, Fukuyama S, Nonami A, Taniguchi K, Matsumoto K, Nakano T, Tsuda M, Matsumura M, Kubo M, et al: Spred-1 negatively regulates allergen-induced airway eosinophilia and hyperresponsiveness. J Exp Med 201: 73-82, 2005.

39. Zhu Q, Xu X, Liu X, Lin J, Kan Y, Zhong Y, Liu F and Xu J: Sodium houttuyfonate inhibits inflammation by blocking the MAPKs/NF- $\kappa \mathrm{B}$ signaling pathways in bovine endometrial epithelial cells. Res Vet Sci 100: 245-251, 2015.
40. Wu H, Zhao G, Jiang K, Chen X, Rui G, Qiu C, Guo M and Deng G: IFN- $\tau$ alleviates lipopolysaccharide-induced inflammation by suppressing NF- $\mathrm{NB}$ and MAPKs pathway activation in mice. Inflammation 39: 1141-1150, 2016.

41. Sheller JR, Polosukhin VV, Mitchell D, Cheng DS, Peebles RS and Blackwell TS: Nuclear factor kappa B induction in airway epithelium increases lung inflammation in allergen-challenged mice. Exp Lung Res 35: 883-895, 2009.

42. Tang N, Ma J, Wang KS, Mi C, Lv Y, Piao LX, Xu GH, Li X, Lee JJ and Jin X: Dihydromyricetin suppresses TNF- $\alpha$-induced $\mathrm{NF}-\kappa \mathrm{B}$ activation and target gene expression. Mol Cell Biochem 422: 11-20, 2016. 